ISSN 2079-9292

www.mdpi.com/journal/electronics

Review

\title{
Anomalous Response in Heteroacene-Based Organic Field Effect Transistors under High Pressure
}

\author{
Ken-ichi Sakai and Jun Takeya \\ Department of Advanced Materials Science, Graduate School of Frontier Sciences, \\ University of Tokyo, 5-1-5, Kashiwanoha, Kashiwa-shi, Chiba 277-8561, Japan; \\ E-Mail: k-sakai@ organicel.k.u-tokyo.ac.jp (K.S.); takeya@k.u-tokyo.ac.jp (J.T.); \\ Tel.: +81-4-7163-3763.
}

Received: 15 February 2014; in revised form: 28 March 2014 / Accepted: 31 March 2014 /

Published: 10 April 2014

\begin{abstract}
Carrier transport properties of organic field effect transistors in dinaphtho[2,3-b:2',3'-f] thieno[3,2-b] thiophene single crystals have been investigated under high pressure. In contrast to the typical pressure effect of monotonic increase in charge transfer rates according to the application of external hydrostatic pressure, it is clarified that the present organic semiconductor devices exhibit nonmonotonic pressure response, such as negative pressure effect. X-ray diffraction analysis under high pressure reveals that on-site molecular orientation and displacement in the heteroacene molecule is assumed to be the origin for the anomalous pressure effects.
\end{abstract}

Keywords: organic field effect transistor; pressure effect; carrier transport; $\mathrm{x}$-ray diffraction analysis

\section{Introduction}

Organic molecular solids, such as organic semiconductors, have been studied intensively due to their considerable scientific and industrial uses. The pristine electronic properties of such material ensures good insulation because of the closed-shell structure of organic molecules, but they can also become electric conducting materials when a charge accumulation layer is formed by the application of an electric field on the surface. The $\pi$-electrons, whose interaction is described by weak van-der-Waals interaction, are responsible for the charge transport, in sharp contrast to those in inorganic semiconductors with a well-defined covalent network for charges to transfer. Hence, organic 
semiconductors have often seemed to provide poor electric conductivity and it appears that the hopping transport mechanism is realized on the basis of localized electronic states. Nevertheless, it has been revealed that some of the organic semiconductor systems show moderate carrier mobility above $1 \mathrm{~cm}^{2} / \mathrm{Vs}$ [1-4] and the charge transport mechanism is dominated by a band transport system described by spatially-distributed Bloch waves similar to ordinary metals, but on the verge of self-localization because of the strong molecular fluctuation [5]. From a microscopic viewpoint, the peculiar shape of molecular orbitals in such compounds causes condensation in the corresponding crystal structure at ambient pressure as a result of naïve balance between mutually attracting London force and Coulomb repulsive force. Therefore, considerable impact is expected with the application of external pressure, not only by reducing molecular distance but also by deforming the crystal structure due to freedom of onsite molecular orientation. This is essentially different from inorganic semiconductors composed of atoms which appear as a spherical mass point. Therefore the application of external pressure is expected to induce a striking change in not only simple reduction of intermolecular molecule distance, but also the deformation of the crystal structure due to the freedom of onsite molecular orientation, and the unanticipated pressure response of electronic properties unlike a simple linear increase in charge transfer rate. Looking at the current progress the electronic industry is making in organic semiconductors, this kind of work becomes necessary for the development of flexible and soft-printed organic devices which is subjected to a bending force behaving like pressure.

In this paper we introduce a method of measuring the effect of hydrostatic pressure on charge carrier conductivity in organic semiconductor crystals, introducing the charge with the application of an electric field on the semiconductor surfaces. In addition to the measurement of common transfer characteristics of drain current as a function of gate voltage shown in former pioneering studies [6,7], we performed four-terminal conductivity measurements to exclude extrinsic influences of metal-to-semiconductor contact resistance [8]. In previous studies, carrier mobility $\mu$ of hydrocarbons, such as rubrene, pentacene, were shown to exhibit a positive pressure response against external pressure $p$, and their pressure coefficient, $\mathrm{d} \mu / \mathrm{d} p$, is extremely larger than used in inorganic semiconductors. Here we focused on the pressure effect of one of heteroacene-based compounds, dinaphtho[2,3-b:2',3'-f]thieno[3,2-b]thiophene (DNTT). It was found that DNTT shows far larger $\mathrm{d} \mu / \mathrm{d} p$ values whose sign can even be negative. The result cannot be explained by the simple model: increase in charge transfer rate with decreasing molecular distance. We note that the pressure effect of molecular solids can be explained by taking into account displacement, rotation and other degrees of freedom originating from molecular shapes. From an x-ray diffraction experiment, we constructed a plausible model of the pressure-induced structural deformation. In combination with the measurement of anisotropic charge transfer and structural studies, a route to study the so-called "structure-property relations" is provided [9]. 


\section{Experimental Section}

\subsection{Preparation of Single-Crystal Organic Semiconductor}

In the present study, platelet-shaped single crystals of DNTT were employed as the channel material. The DNTT single crystals were prepared by the physical vapor transport method [10]. After purification of crude powder samples supplied from Nipponkayaku Co. by vaporizing at $285{ }^{\circ} \mathrm{C}$ and coarse crystallization at $213{ }^{\circ} \mathrm{C}$ in a two-zone tube furnace under Ar gas flow (>99.999\% for gas purity and $200 \mathrm{ccm}$ for flow rate, respectively), the coarse crystals are slowly recrystallized at the same temperature in three days.

\subsection{Carrier Transport Measurement under High Pressure}

In order to realize stable and reproducible measurement under high pressure, device structure and pressure cells were newly developed. The charge transport measurements are performed by the four-terminal technique at room temperature to obtain the information about intrinsic charge transfer properties of channel material. All the components of the measured devices are fabricated by polymer materials except for electrodes so that nearly homogeneous shrinkage by pressurization is realized. The field-effect transistor devices with the top-contact and bottom-gate structure are fabricated as follows. A patterned gate electrode of $20 \mathrm{~nm}$ thickness is vapor-deposited through a shadow mask on a polyethylene naphthalete (PEN) substrate. A gate-insulating layer of CYTOP (Asahi Glass Co., Tokyo, Japan) is formed by spin-coating and subsequent heat treatment at $140{ }^{\circ} \mathrm{C}$ for $15 \mathrm{~h}$. A single-crystal DNTT flake with a thickness of less than $1 \mu \mathrm{m}$ is carefully taken and laminated on the position of the gate electrode. DNTT samples with well-defined crystallographic shape are chosen for experiments so that anisotropic effect of transport properties can be discussed (Figure 1). Then Source-drain electrodes and four voltage terminals are formed by vapor deposition of 2,3,5,6-tetrafluoro-7,7,8,8tetracyanoquinodimethane and over-deposited gold layers through a shadow mask to the thickness of $1 \mathrm{~nm}$ and $20 \mathrm{~nm}$, respectively. Carbon past is used to cover the step edges of electrodes on the periphery of DNTT single crystal. The central channel area is finally shaped into Hall-bar structure by laser etching process and just on the top of the gate electrode. The channel size is $100 \mu \mathrm{m}$ in width and $150 \mu \mathrm{m}$ in length, respectively. The distance between adjacent voltage terminals along the channel direction is $50 \mu \mathrm{m}$. Whole device structure is illustrated in Figure 2a,b. The device is covered with a-few- $\mu \mathrm{m}$ thick parylene for passivation and the completely and then completely filled in epoxy (STYCAST 1266) for the mechanical stability. The devices are introduced in a standard $\mathrm{Cu}-\mathrm{Be}$ pressure cell and Fluorinert FC70/77 is used for the pressurizing medium (Figure 2c).

In the present study pressure dependence of the four-terminal carrier mobility at each pressure $\mu(P)$ is taken into account for quantitative argue of pressure effect of the charge transport. $\mu$ is defined as

$$
\mu=\frac{1}{C} \frac{\partial \sigma}{\partial V_{G}}
$$

where $C$ is capacitance of the CYTOP gate insulator in a unit area, $\sigma$ is the four-terminal sheet conductivity and $V_{G}$ is the applied gate voltage. $C$ is modified as a Pressure-variable number $C(P)$ which is identified in our previous study; we estimated dependent carrier mobility under each pressure 
by precise Hall-effect measurement using rubrene single-crystal Field-Effect-Transistors (FETs), so that $C(\mathrm{P})$ turned out to change by $10 \%$ at $0.5 \mathrm{GPa}$ [8]. Carrier mobility is estimated at $V_{G}=-100 \mathrm{~V}$, where field-induced carriers are accumulated at the bottom of the conduction band into avoid contribution of trap-involved pressure effect in the subthreshold regions as is investigated in rubrene single-crystal transistors.

Figure 1. A photograph of a single crystal dinaphtho[2,3-b:2',3'-f]thieno[3,2-b]thiophene (DNTT) platelet. Crystalline direction for the $a$ and $b$ axes are indicated by white arrows [9].

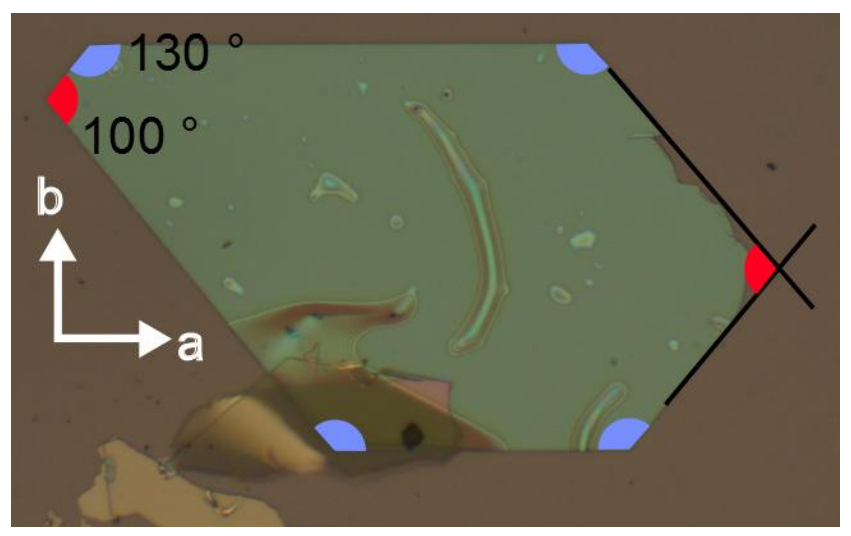

Figure 2. (a) Side view and (b) top view of device structure; (c) Schematic illustration of pressure cell used for the present measurement.

(a)

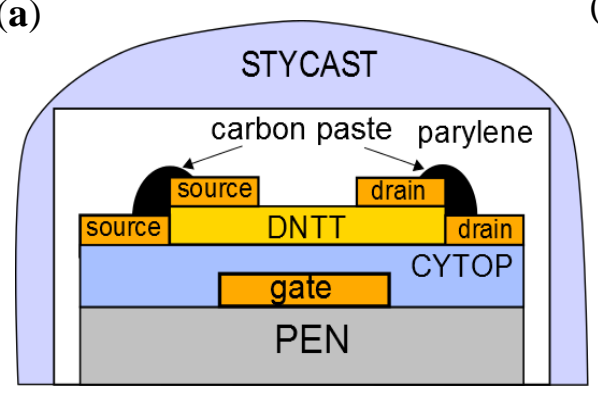

(b)

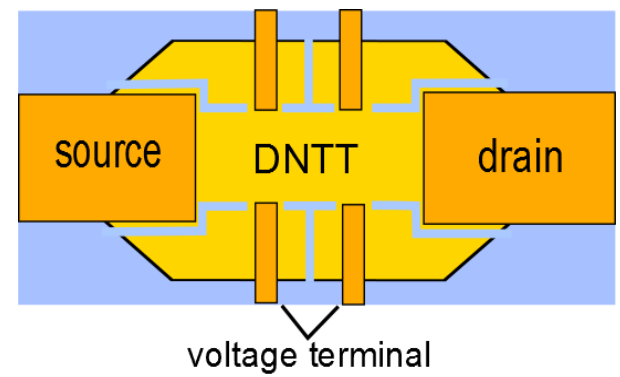

(c)

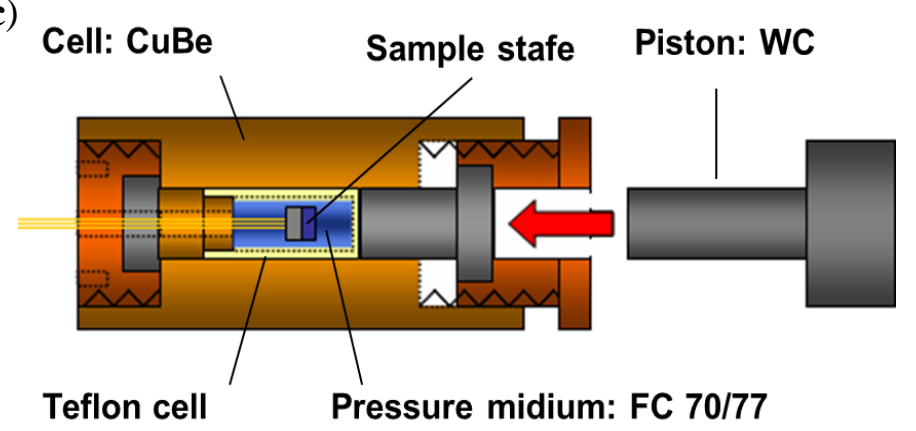

\subsection{Structural Analysis}

X-ray diffraction (XRD) experiments were carried out using well-purified DNTT powder by a synchrotron radiation source on the beamline BL10XU at SPring-8 in order to have an idea of change 
in unit cells in high pressure. The sample is introduced into a diamond anvil cell with the pressure medium (FC70/77). Applied pressure is measured by a simultaneous ruby fluorescence measurement. The wavelength of X-ray is $0.41413 \AA$. The diffraction patterns were obtained with an imaging plate detector as a function of $2 \theta$ ( $\theta$ is an incident angle of $\mathrm{X}$-ray). Here we should pay attention that deformation of unit cell observed in powder X-ray diffraction experiment is not precisely consistent with that in our transistors. However, they are somehow comparable due to the fact that the anisotropic behavior of lattice constants in rubrene single crystal under high pressure is consistent with that of carrier mobility [11].

\section{Results and Discussion}

The unit cell of DNTT is monoclinic, meaning that there are four lattice parameters, lattice constants along $a, b$, and $c$ axes and an angle $\beta$, defined as crossing angle of $a$ and $c$ axes. The XRD patterns as a function of $2 \theta$ at each pressure point are shown in Figure 3. No disappearance and emergence of peaks in pressurizing process is observed, indicating that there is no structural phase transition up to $2 \mathrm{GPa}$. Every peak shifts toward larger angle, indicating the overall shrinkage of unit cell. We choose four distinctive peaks assigned to (100), (003), (020) and (111) so that the four lattice parameters can be identified. Note that the result is consistent with the positions of all over visible peaks.

Figure 3. XRD patterns for pressurized powder DNTT. Red, blue, green and dark arrows indicate (100), (003), (020) and (11-1) reflection peaks, respectively [9].

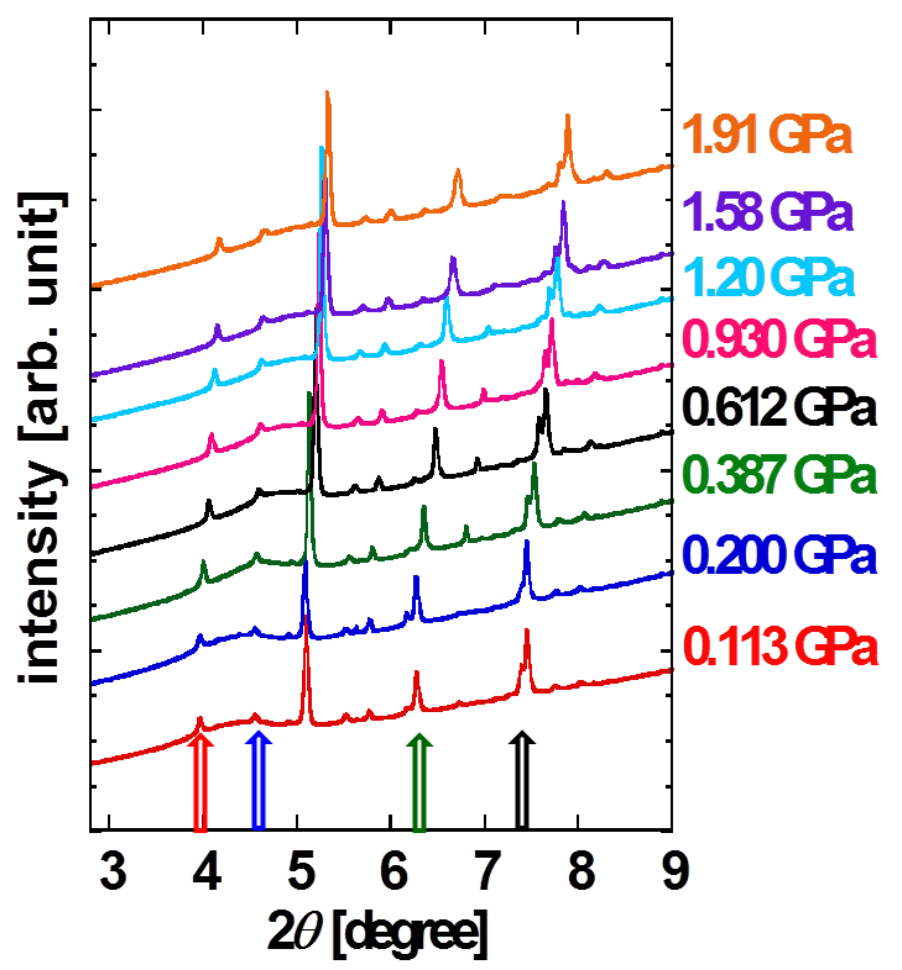

Figure $4 \mathrm{a}, \mathrm{b}$ indicate the variations of these parameters as a function of pressure. It was found that the lattice parameters $a, c$ and $\beta$ shows rapid variation at the lower pressure range (approximately less than $0.2 \mathrm{GPa}$ ) and then moves to more moderate pressure dependency at higher pressure range, in 
contrast to the variation of $b$ showing an almost constant rate. For further discussion consider the molecular rearrangement in the process of pressurization. Figure $4 \mathrm{c}$ shows the ratio of compressibility in $a\left(\kappa_{a}\right)$ and $b\left(\kappa_{b}\right)$ directions. Here the compressibility is defined as the differential coefficient estimated from two adjacent measurement points,

$$
\kappa(P)=\frac{1}{L\left(P_{i}\right)} \frac{L\left(P_{i}\right)-L\left(P_{j}\right)}{P_{i}-P_{j}}
$$

where $P_{i}$ and $P_{j}$ are two adjacent pressure points $\left(P_{i}>P_{j}\right) . L\left(P_{i}\right)$ and $L\left(P_{j}\right)$ are the lattice constants evaluated at each pressurized condition and $P$ is the average value between $P_{i}$ and $P_{j}$, defined as $\left(P_{i}+P_{j}\right) / 2$ for the sake of convenience since lattice constants plotted in present paper are discrete value; therefore $x$-values of data points in Figure 4a,c are deviated.

Figure 4. Pressure dependence of (a) lattice constants for three crystallographic directions and (b) $\beta$, defined as the intersection angle between $a$ and $c$ axes; (c) Compressibility ratio between $a$ and $b$ axes as a function of applied pressure; (d) Schematic diagram of suggested molecular rearrangement in $a b$ plane. The two pictures depict the molecular rearrangements in case of shrinkage along the $a$ and $b$ directions, respectively. Red and blue arrows indicate the rotation direction; (e) Crystal structure in $a c$ plane. Dark and light molecular images and arrows indicate molecules located on the front and reverse side and corresponding displacement direction in pressurization process, respectively [9].
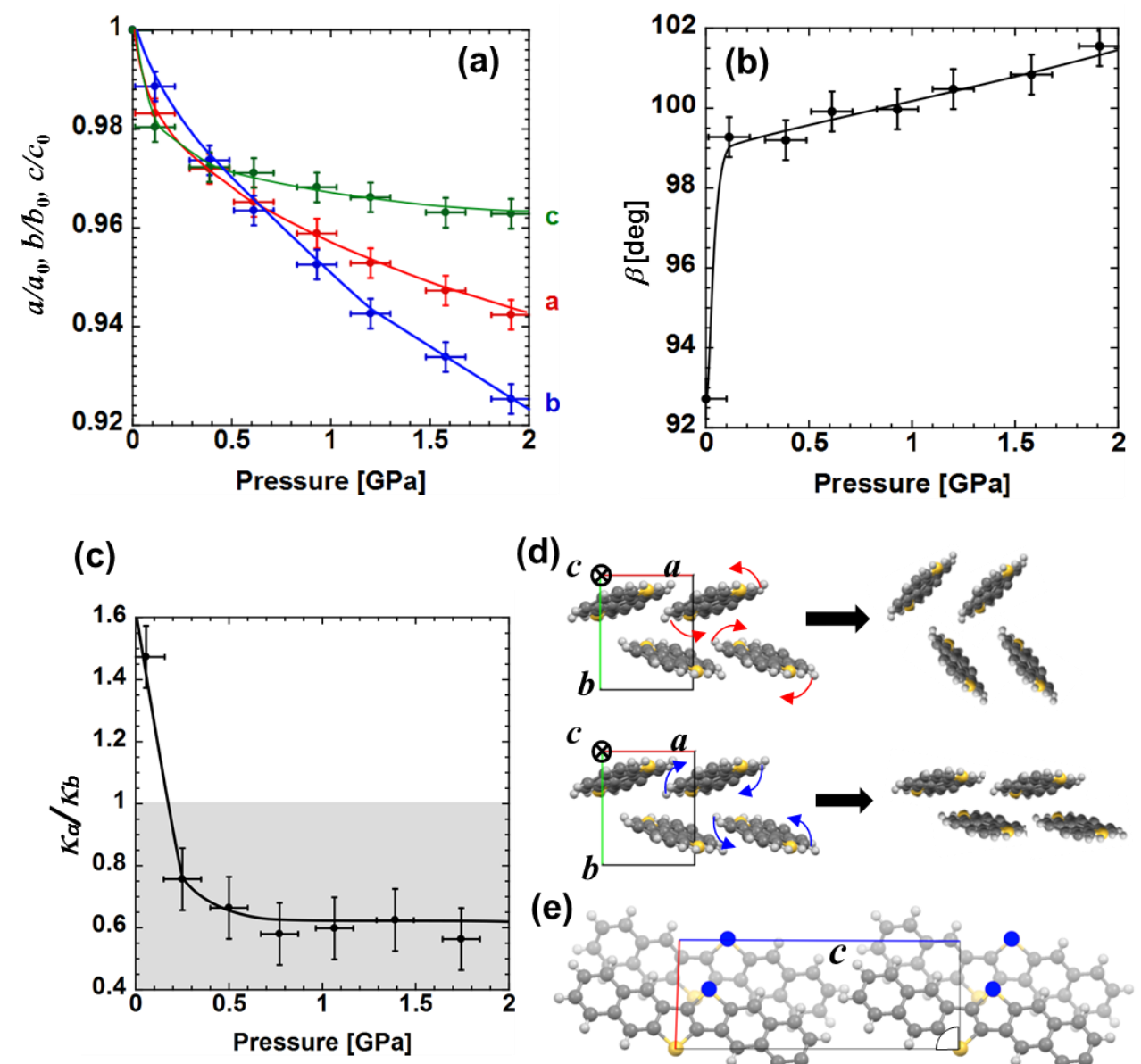

(d)

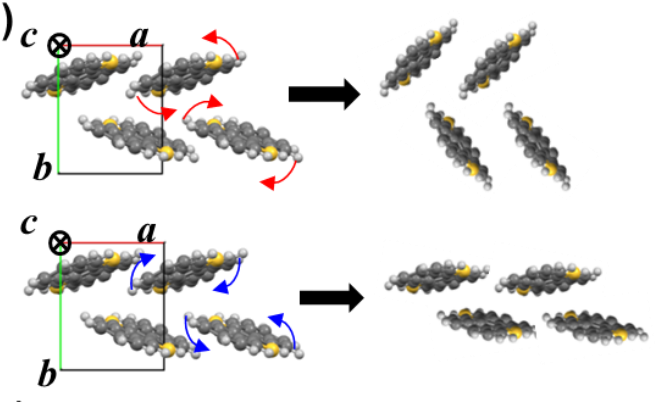

(e)

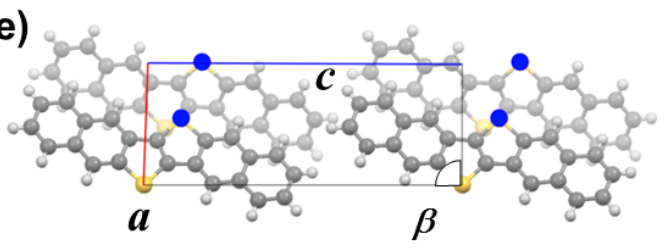


Let us focus on the discussion of the pressure effect below approximately $0.2 \mathrm{GPa}$. Within this pressure range $\kappa_{a} / \kappa_{b}$ is larger than 1 , suggesting that the deformation of unit cell in $a b$ plane is dominated by the shrinkage along $a$-axis. In this case molecules are supposed to be rearranged with concomitant rotation indicated by the curved red arrows in Figure $4 \mathrm{~d}$. For a higher pressure range, the unit cell starts to shrink along $b$-axis since $\kappa_{a} / \kappa_{b}<1$, in which the molecules are supposed to start onsite rotation along the opposite direction to that in the lower pressure range (curved blue arrows in Figure 4d). Note that the relationship between the dominant shrinkage direction of unit cell and the corresponding molecular rearrangement mentioned above is consistent with structural change experimentally observed in the oligoacene system, such as anthracene, pentacene and tetracene under high pressure [12,13].

It is noted that similar pressure dependences are shown among $a, c$ and $\beta$ : drastic change in lower pressure region and the following slow change in higher pressure region (Figure 4d). To approach this experimental result let us take into account the molecular rearrangement in ac plane which is characterized by these three lattice parameters. As an external mechanical force is added intermolecular interaction is enhanced due to the access of nearest-neighbor molecules. The $\pi$-electrons tend to be localized on electro-negative sulfur atoms as compared to carbon and hydrogen sites [14], in addition to the effect of spatially large occupation of the $3 p_{\mathrm{z}}$ orbitals on sulfur atoms. Therefore it is expected that intermolecular interaction of nearest-neighbor DNTT molecules are highly dominated by effects between sulfur atoms of different molecules. As the interaction between nearest-neighbor sulfur atoms marked by blue circles in Figure 4e becomes large with increasing pressure, the adjacent molecules tend to displace along $c$-axis to reduce the energy loss of Coulomb repulsion. Because sulfur-to-sulfur interaction is pronounced along $a$-axis, both $c$ and $\beta$ parameters exhibit drastic change under relatively low pressure in the region of $\kappa_{a} / \kappa_{b}>1$ where shrinkage of $a$ is dominant, while the values are less sensitive to pressure when shrinkage onn the $b$-axis is dominant at higher pressure. The greater sulfur-to-sulfur interaction along the $a$-axis than the $b$-axis is also consistent with higher carrier mobility on the $a$-axis compared to that along the $b$-axis. Note that the change in $\beta$ up to $10^{\circ}$ at around $2 \mathrm{GPa}$ contrasts that observed in sulfur-free oligoacenes in which only a few degrees of deviation up to $10 \mathrm{GPa}$ is observed for angles, so called $\alpha, \beta$ and $\gamma[12,13]$.

Pressure effects of carrier mobility in DNTT-FETs are measured along two distinctive crystallographic directions, $a$ - and $b$-axes. Transport and output characters and carrier conductivity as a function of gate voltage are indicated in Figure 5 for each axis. For quantitative discussion of pressure effects on carrier transport properties of DNTT-FETs, let us introduce the increasing rate defined as

$$
\frac{\Delta \mu(P)}{\mu(0)}=\frac{[\mu(P)-\mu(0)]}{\mu(0)}
$$

where $\mu(P)$ and $\mu(0)$ are four-terminal mobility at a certain pressure $P$ and that in the ambient condition. Note that estimated carrier mobility along each axis in ambient conditions is $2.1 \mathrm{~cm}^{2} / \mathrm{Vs}$ for $a$-axis and $1.3 \mathrm{~cm}^{2} / \mathrm{Vs}$ for $b$-axis respectively. This anisotropic value is in agreement with a previous report [15]. The pressure dependence of carrier mobility is shown in Figure 6 at the drain bias voltage $V_{D}$ of $-10 \mathrm{~V}$. In the present study, the reproducibility of carrier mobility against compression and decompression is confirmed. Simultaneous plots of pressure-dependent carrier mobility of rubrene [8] and pentacene [16] devices reveal that carrier transport of DNTT is much more sensitive to 
pressurization and, furthermore, shows the nonmonotonous pressure response characterized by the appearance of peak and valley at $0.15 \mathrm{GPa}$ on the $a$-axis and $b$-axis devices, respectively. Note that this is a quite different feature in contrast to two other hydrocarbon FETs with simple linear responses.

Figure 5. (a) Transport and (b) output properties of DNTT-FET for $a$-axis conducting direction; (c) transport properties of DNTT-FET for $b$-axis conducting direction. Photographs for each devices are inserted in (a) and (c); (d) four-terminal conductivity in $a$ and $b$-axes at ambient condition. The estimated carrier mobility is $2.1 \mathrm{~cm}^{2} \mathrm{~V}^{-1} \mathrm{~s}^{-1}$ for $a$-axis and $1.3 \mathrm{~cm}^{2} \mathrm{~V}^{-1} \mathrm{~s}^{-1}$ for $b$-axis at $\mathrm{VG}=-100 \mathrm{~V}$, respectively [9].
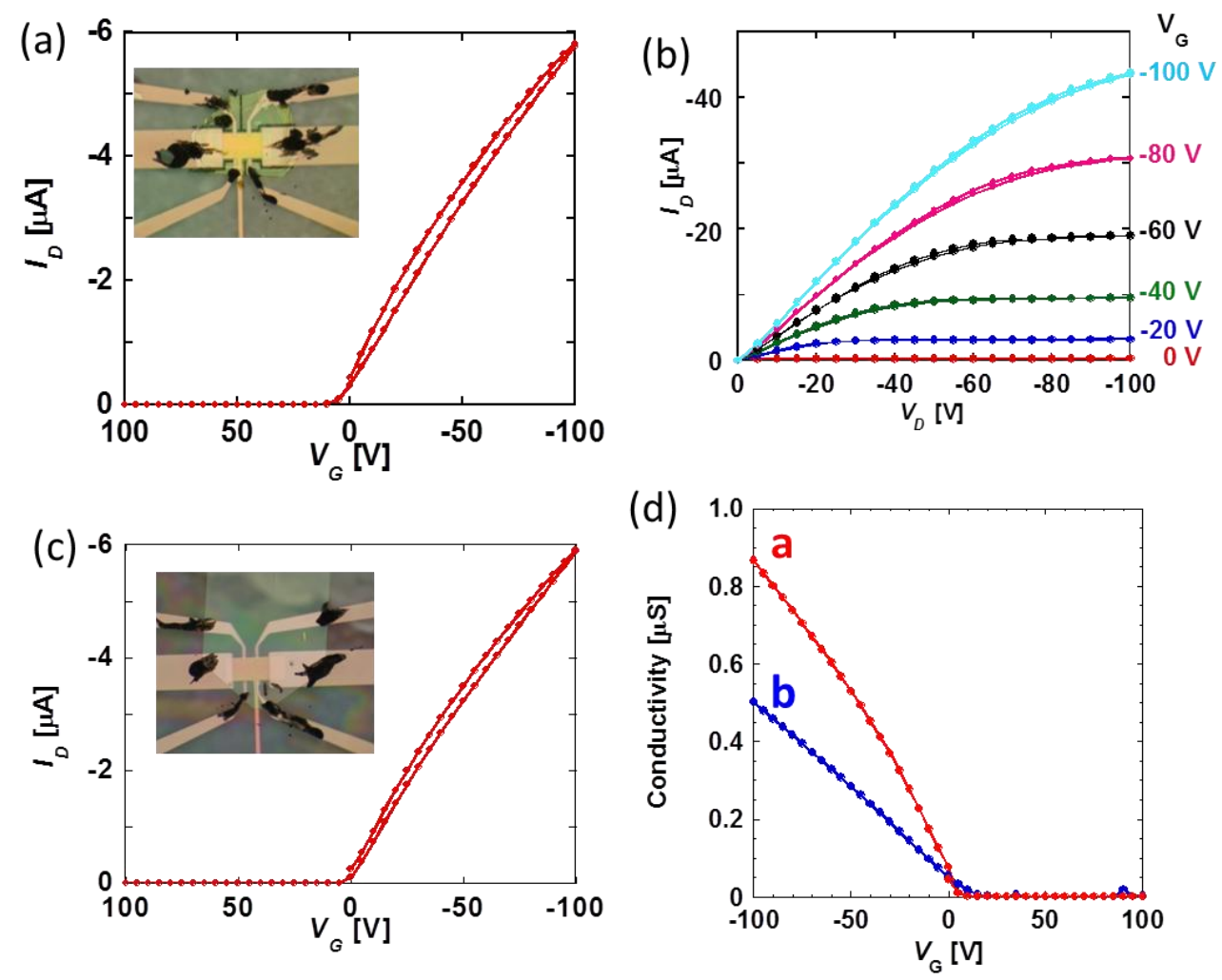

Figure 6. Pressure effect on DNTT-FET for $a$ and $b$-axes [9]. Those for pentacene [16] and rubrene [8] devices are also plotted for comparison. Open and closed circles indicate the data obtained in compression and decompression processes.

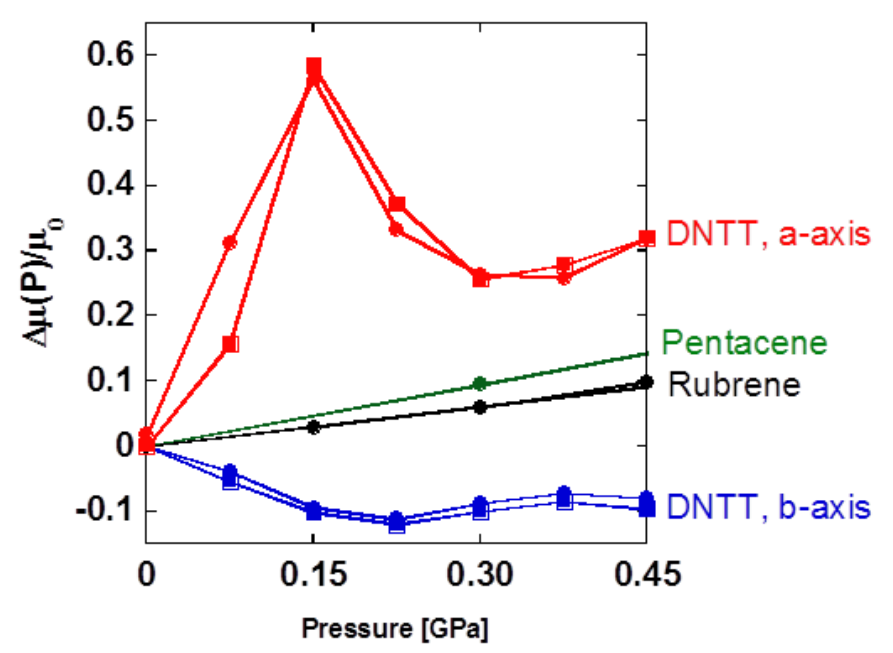


To assist in understanding this phenomenon, it is good to associate this problem with the structural variation by external pressure as shown in Figure 4. The appearance of a peak in $\Delta \mu(P) / \mu(P)$ can be taken that the sign of the derivative $\mathrm{d} \mu / \mathrm{d} P$ for each axis is converted at $0.15 \mathrm{GPa}$, which coincides with the crossover point of $\kappa_{a} / \kappa_{b}=1$, representing a simultaneous change in the anisotropic deformation of unit cell at the same pressure. Therefore the anomalous pressure response of carrier mobility on DNTT-FET seems to be connected with the change in molecular arrangement mentioned above. When $p<0.15 \mathrm{GPa}$, our results suggest that the molecules are likely to rearrange so that face-to-face $\pi-\pi$ stacking is enhanced along the $a$-axis, which leads to the increase in carrier mobility on the $a$-axis and decrease on the $b$-axis. In contrast, when $p>0.15 \mathrm{GPa}$, the structural change has the opposite effect, so that carrier mobility decreases on the $a$-axis and increases on the $b$-axis, respectively. Therefore the peak structure results from crossover between the two distinct molecular rotations associated with pressurization. At a higher pressure than $0.30 \mathrm{GPa}$, a slight increase in carrier mobility on the $a$-axis can be seen due to the predominance of the normal pressure effect from the increase in density of molecular packing. Note that the contribution of molecular rearrangement is suppressed in the profile of $\Delta \mu(P) / \mu(P)$ in this pressure region. We have demonstrated the relationship of carrier transport properties and the structure of DNTT-FETs via their pressure response. However, for further elucidation of structure-property relations of pressure-sensitive organic semiconductors, crystal structure analysis under high pressure should be completed so that we complement the detailed information about the molecular rearrangement. The magnitude of pressure coefficient is up to $3.8 \mathrm{GPa}^{-1}$ along the $a$-axis and is extremely large below $0.15 \mathrm{GPa}$ as compared to those of rubrene $\left(0.2 \mathrm{GPa}^{-1}\right)$ and pentacene $\left(0.4 \mathrm{GPa}^{-1}\right)$, which is already one order larger than typical values for inorganic materials [17]. In conjunction with the significant contribution of the sulfur-to-sulfur interaction which causes peculiar displacement in the $a c$ plane, the presence of sulfur atoms in DNTT is responsible for the anomalous pressure dependence in carrier mobility, indicating the significant contribution of $3 p_{\mathrm{z}}$ orbital on sulfur to the charge transport in the semiconductors and to the device performance. It is highly expected that the more efficient charge transfer on the $a$-axis is realized in DNTT FETs by pressurization, due to the enhancement of orbital overlap, and especially the presence of more spatially-spread $3 p_{z}$ orbital on sulfur atoms than those for carbon and hydrogen, the main components of rubrene and pentacene.

\section{Conclusions}

Charge transport properties and structures of DNTT have been investigated under high pressure. The crossover of on-site molecular rotation and simultaneous anisotropic deformation of unit cells have been observed. It is suggested that access to nearest-neighbor sulfur atoms as a result of pressurization is responsible for both in order to suppress the repulsive electrostatic interaction between negatively-charged sulfur atoms. Also, the corresponding pressure effect of charge transport on DNTT-FETs can be well-explained by the structural change, indicating the successful observation of so-called structure-property relations. Furthermore the giant pressure effect with extreme pressure response up to $3.8 \mathrm{GPa}^{-1}$ is attributed to the widely-distributed $3 p_{\mathrm{z}}$ orbital localized on sulfur atoms. Through this study, we have clarified that the presence of sulfur atom plays an important role for the realization of anomalous and giant pressure effect observed. It is noteworthy that nonmonotonous 
evolution of structural change and charge transport is attributed to the specific molecule-to-molecule interaction due to the peculiar molecular shape, in sharp contrast to the case of inorganic semiconductors which can be seen as assemblies of more symmetric atoms. These experimental observations evidence how heteroelement affects the properties of heteroacene-based molecular compounds. It is demonstrated that investigating pressure effects of organic semiconductors is a powerful method to clarify the relationship between charge transport mechanisms and microscopic molecular alignments. The knowledge obtained will assist in designing new compounds and practical devices, such as flexible and soft printed devices in which a mechanical force is applied in the bending process.

\section{Acknowledgments}

Authors thank Y. Okada, T. Uemura, S. Kitaoka and J. Tsurumi for the assistance of device experiment, Y. Ohishi and A. Fujiwara for powder X-ray diffraction experiment at SPring-8 and K. Takimiya and Nippon Kayaku Co., Ltd. for supplying DNTT sample powders. This work is financially supported by Grant-in-Aid for Scientific Research (Grants Nos. 22245032 and 236860050) from MEXT in Japan.

\section{Conflicts of Interest}

The authors declare no conflict of interest.

\section{References}

1. Takeya, J.; Tsukagoshi, K.; Aoyagi, Y.; Takenobu, T.; Iwasa, Y. Hall Effect of Quasi-Hole Gas in Organic Single-Crystal Transistors. Jpn. J. Appl. Phys. 2005, 44, L1393-L1396.

2. Podzrov, V.; Menard, E.; Rofers, J.A.; Gershenson, M.E. Hall Effect in the Accumulation Layers on the Surface of Organic Semiconductors. Phys. Rev. Lett. 2005, 95, 226601.

3. Takeya, J.; Kato, J.; Hara, K.; Yamagishi, M.; Hirahara, R.; Yamada, K.; Nakazawa, Y.; Ikehata, S.; Tsukagoshi, K.; Aoyagi, Y.; et al. In-Crystal and Surface Charge Transport of Electric-Field-Induced Carriers in Organic Signle-Crystal Semiconductors. Phys. Rev. Lett. 2007, 98, 196804.

4. Yamagishi, M.; Soeda, J.; Uemura, T.; Okada, Y.; Takatsuiki, Y.; Nishikara, T.; Nakazawa, Y.; Doi, I.; Takimiya, K.; Takeya, J. Free-electron-like Hall effect in high mobility organic shin-film transistors. Phys. Rev. B 2010, 81, 161306(R).

5. Uemura, T.; Yamagishi, M.; Soeda, J.; Takatsuiki, Y.; Okada, Y.; Nakazawa, Y.; Takeya, J. Temperature dependence of the Hall effect in pentacene field-effect transistors: Possibility of charge decoherence induced by molecular fluctuations. Phys. Rev. B 2012, 85, 035313.

6. Rang, Z.; Natethan, M.I.; Ruden, P.P.; Chesterfield, R.; Daniel Frisbie, C. Hydrostatic pressure dependence of organic thin-film transistors current versus voltage characteristics. Appl. Phys. Lett. 2004, 85, 5760.

7. Rang, Z.; Natethan, M.I.; Ruden, P.P.; Podzorov, V.; Gershenson, M.E.; Newman, C.R.; Frisbie, C.D. Hydrostatic pressure dependence of charge carrier transport in single-crystal rubrene devices. Appl. Phys. Lett. 2005, 86, 123501. 
8. Okada, Y.; Sakai, K.; Uemura, T.; Nakazawa, Y.; Takeya, J. Charge transport and Hall effect in rubrene single-crystal transistors under high pressure. Phys. Rev. B 2011, 84, 245308.

9. Sakai, K.; Okada, Y.; Kitaoka, S.; Tsurumi, J.; Ohishi, Y.; Fujiwara, A.; Takimiya, K.; Takeya, J. Anomalous Pressure Effect in Heteroacene Organic Field-Effect Transistors. Phys. Rev. Lett. 2013, 110, 096603.

10. Klok, C.; Simpkins, P.G.; Siegrist, T.; Laudise, R.A. Physical vapr growth of centimeter-sized crystals of $\alpha$-hexathiophene. J. Cryst. Growth 1997, 182, 416.

11. Okada, Y.; Sakai, K.; Kitaoka, S.; Miwa, K.; Ohishi, Y.; Takeya, J. Charge Transport and Srystal Structure in Rubrene Single-Crystal Transistors under High Pressure Proceeding of the $67^{\text {th }}$ Annual Meeting of Physical Society of Japan, Nishinomiya, Hyogo, Japan, 19 March 2012.

12. Oehzelt, M.; Resel, R.; Nakayama, A. High-pressure structural properties of anthracene up to $10 \mathrm{GPa}$. Phys. Rev. B 2002, 66, 174104.

13. Oehzelt, M.; Aichholzer, A.; Resel, R.; Heimel, G.; Venuti, E.; Della Valle, R.G. Crystal structure of oligoacenes under high pressure. Phys. Rev. B 2006, 74, 104103.

14. S-Carrera, R.S.; Atahan, S.; Schrier, J.; A-Guzik, A. Theoretical Characterization of the Air-Stable, High-Mobility Dinaphtho[2,3-b:2',3'-f]thieno[3,2-b]thiophene Organic semiconductor. J. Phys. Chem. C 2010, 114, 2234-2340.

15. Uno, M.; Tominari, Y.; Yamagishi, M.; Doi, I.; Miyazaki, E.; Takimiya, K.; Takeya, J. Moderately anisotropic field-effect mobility in dinaphtho[2,3-b:2',3'-f]thiopheno[3,2-b] thiophenes single-crystal transistors. App. Phys. Lett. 2009, 94, 223308.

16. Sakai, K.; Okada, Y.; Kitaoka, S.; Tsurumi, J.; Takeya, J. Proceeding of the $67^{\text {th }}$ Annual Meeting of Physical Society of Japan, Nishinomiya, Hyogo, Japan, 19 March 2012.

17. Hutson, A.R.; Jayaraman, A.; Coriell, A.S. Effects of High Pressure, Uniaxial Stress, and tempareture on the Electrical Resistibity of $n$-GaAs. Phys. Rev. 1967, 155, 786-796.

(C) 2014 by the authors; licensee MDPI, Basel, Switzerland. This article is an open access article distributed under the terms and conditions of the Creative Commons Attribution license (http://creativecommons.org/licenses/by/3.0/). 